\title{
Some Activities of MISSE 6 Mission
}

\author{
Narasimha S. Prasad* \\ NASA Langley Research Center, 5 N. Dryden St., MS 468, Hampton VA, 23681
}

\begin{abstract}
The objective of the Materials International Space Station Experiment (MISSE) is to study the performance of novel materials when subjected to the synergistic effects of the harsh space environment for several months. In this paper, a few laser and optical elements from NASA Langley Research Center (LaRC) that have been flown on MISSE 6 mission will be discussed. These items were characterized and packed inside a ruggedized Passive Experiment Container (PEC) that resembles a suitcase. The PEC was tested for survivability due to launch conditions. Subsequently, the MISSE 6 PEC was transported by the STS-123 mission to International Space Station (ISS) on March 11, 2008. The astronauts successfully attached the PEC to external handrails and opened the PEC for long term exposure to the space environment. The plan is to retrieve the MISSE 6 PEC by STS-128 mission in August 2009.
\end{abstract}

Keywords: MISSE 6, Space Qualification, Laser components, International Space Station, STS-123.

\section{INTRODUCTION}

The goal of MISSE program is to evaluate the performance, stability, and long-term survivability of materials and components planned for use by NASA, Department of Defense (DOD), other federal agencies and private entities on future Low Earth Orbit (LEO), synchronous orbit, and interplanetary space missions. The study of combined effects of radiation, ultraviolet (UV) light and atomic oxygen in space due to long term exposures will help in developing space qualifiable elements for future space missions. In-situ space testing is critical since it is difficult and not economical to conduct these studies inside simulated terrestrial facilities. The development of new generations of materials will allow our nation to continue maintain technological superiority related to space endeavors.

NASA's Langley Research Center, Hampton, Virginia has managed the MISSE projects till recently. Other NASA Centers participating in MISSE project include Glenn Research Center, Cleveland; Goddard Space Flight Center, Greenbelt, Maryland, Johnson Space Center, Houston, Texas, the Jet Propulsion Laboratory, Pasadena, California, and Marshall Space Flight Center, Huntsville, Alabama.. Several DOD and industry partners including the Boeing Company have been involved with this effort. MISSE program is a direct successor of the Mir Environmental Effects Payloads (MEEP) that were attached for over a year to the Mir Docking Module of the space station Mir between shuttle flights STS-76 and STS-86 ${ }^{1}$.

\section{AN OVERVIEW OF MISSE PROGRAM}

MISSE is a series of experiments and so far, five MISSE missions have been successfully completed. More than 1500 samples have been tested on the MISSE project. Samples include chemicals, sensor devices, opto-mechanical elements, polymers, coatings, and biological materials and species, composites. The MISSE project has also provided educational opportunities for students. MISSEs 3 and 4 have transported approximately 8 million basil seeds for science experiments of children to stimulate interest in space science.

A suitcase shaped rugged box known as Passive Experiment Containers (PECs) is used to transport the selected materials to and from the ISS. PECs were originally developed and used by NASA's Langley Research Center, Hampton, Virginia, for ISS Phase I Risk Mitigation Program experiments conducted on the Russian Mir space station. Specific steps required for transporting materials include specimen preparation, fixing specimens inside a holder,

*narasimha.s.prasad@nasa.gov; Phone 757-864-9403; Fax 757-864-8828. 
integration of specimen holder on a tray, integration of trays in a PEC and integration of PEC inside a carrier for ease of transport. All these tasks are carried out in clean room environment.

The PEC is tested for survivability under launch conditions by subjecting it to appropriate shock and vibration environment. Subsequent to several months of space exposure, the PEC will be transported back to the Earth to undergo tests. The resulting test characteristics when compared with original characteristics before launch will provide an understanding of their survivability in space conditions. Furthermore, it will provide insights into required space qualification processes for future space applications.

During space walk, also known as extra-vehicular activity (EVA) (i.e., when an astronaut works outside of a spacecraft), the PECs are attached to the handrails or at a specific location that is exposed to space environment. Two possible mounting positions known as ram and wake are available. The materials in the ram side will be predominantly subjected to atomic oxygen environment where as those materials in the wake side will mostly undergo UV exposure. After exposure in space, MISSE PEC is retrieved in the same manner as it was deployed. The material samples are tested in laboratory conditions to see if they still possess their unique properties needed to complete space missions. MISSE PECs have active and passive detectors to give a time-history reading, or a reading of what happened to the materials at certain points in time. Back on the ground, tests will be conducted to determine the effects of its exposure for several months in space. The passive detectors report a cumulative measure of the following environments that the test specimens are exposed to namely UV exposure, atomic oxygen exposure, molecular contamination, tray temperature and man-made debris.

Terrestrial laboratories may provide limited environmental test conditions while in space, the components are simultaneously exposed to several conditions which normally are difficult to simulate. In many cases, the combined effect of being exposed to all of the environmental elements at once would provide insights into ruggedization for future space applications. The details of MISSE 1 to MISSE 5 missions can be found at NASA's MISSE webpage ${ }^{2}$. In this paper, an overview of MISSE 6 mission followed by laser based optical components sent by NASA LaRC is discussed.

\section{SELECTED ITEMS FOR MISSE 6 MISSION}

Laser Remote Sensing Branch in Systems Engineering Directorate (SED) at NASA Langley Research Center is involved with forefront research of novel technologies including space based lidars suitable for future space exploration missions. Among several specimens from NASA Langley, several optical and laser related components were included in the MISSE 6 mission $^{3}$. MISSE 6 package has two sections namely MISSE 6A and MISSE 6B corresponding to ram and wake sides, respectively. The components from SED were located on the MISSE 6B section. In the following sections, a brief summary of these specimens are discussed.

\subsection{Optical elements for MISSE 6 mission}

Table 1 lists novel materials and components that are of considerable interest for various NASA applications. These elements have been characterized in the laboratory and were included for MISSE 6B platform specifically for UV and radiation exposure tests. The impact of space elements on these items for long term operational stability and survivability have to be evaluated for risk mitigation purposes. The data gathered under MISSE 6 mission will play a significant role in further development of these items for space applications. A brief description on significance of items listed in Table 1 is as follows:

Table 1. Optical specimens and their specifications

\begin{tabular}{|c|c|c|c|c|c|}
\hline Na & TETn & 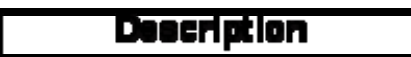 & Emonolone & एजil: & K:m \\
\hline 1 & $\begin{array}{l}\text { Carremle Yut } \\
\text { raos) }\end{array}$ & 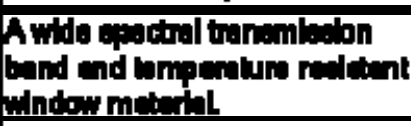 & 12x16xi & $\mathrm{mm}^{\mathrm{a}}$ & Polletad oplces mated \\
\hline 2 & E:Porn & 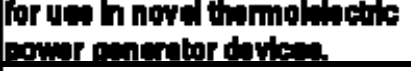 & $12 \times 12.96$ & $\mathrm{~mm}^{2}$ & 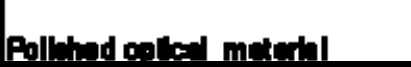 \\
\hline 3 & Leon Dhdo & TO-3 Pechenen & espopas & $\min 3$ & 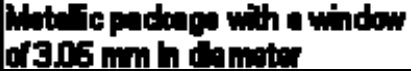 \\
\hline 4 & 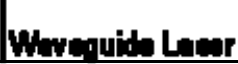 & 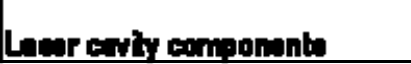 & $\operatorname{sextax} 2$ & $\mathrm{~mm}^{3}$ & 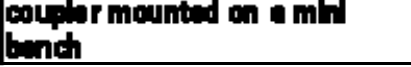 \\
\hline
\end{tabular}


Ceramic Yttria: Ceramic Yttria is being developed for novel UV laser applications under NASA SBIR Phase II program. In this program, optical quality Nd ion doped ceramic Yttria material of appreciable size has been successfully developed using unique material processing techniques ${ }^{4}$. Yttria has spectral transmission range starting from $200 \mathrm{~nm}$. Greater than $99 \%$ transmission coefficient over visible and near-IR spectral band has been measured. This material is anticipated to play a significant role in the development of compact short pulsed high pulse energy lasers for remote sensing of trace gases, predominantly ozone, as well as in chem-bio sensing applications. One of the secondary applications of Yttria is in optical windows for crew launch vehicles (CLVs) and crew exploration vehicles (CEVs). This material has potential for use as windows in high temperature $(>2400 \mathrm{C})$ and high pressure $(>30,000 \mathrm{psi})$ environments. Space crafts will be subjected to these conditions during re-entry into the Earth's atmosphere.

Bismuth doped Lead Telluride (Bi:PbTe) and Silver doped Antimony telluride (Ag: $\left.\mathbf{S b}_{2} \mathbf{T e}_{3}\right)$ : These two semiconductor materials are being investigated for the development of improved efficiency, all solid-state, and compact thermoelectric power generators and cooling elements using nanotechnology. This effort was partially supported under NASA LaRC's funding. Bi:PbTe as n-type and $\mathrm{Ag}: \mathrm{Sb}_{2} \mathrm{Te}_{3}$ as p-type have been successfully tested. So far, promising results have been obtained in regard to their figure-of-merit characteristics. Following material development, fabrication of a prototype thermo electric power generator device has been completed and is now being characterized. Thermoelectric power generators using various heat sources including solar radiation, radioactive isotopes, and waste heat can generate modest electrical power over long durations and hence are attractive for lunar, Mars and other planetary missions. Unlike solar cells, thermoelectric power generators can operate under sealed conditions thereby avoiding damages from extraneous sources.

Semiconductor Laser Diode: The laser diode of interest operates around 2 micron wavelength region. Besides eyesafe optical communications, this laser diode can be used for trace gas detection specifically Carbon dioxide $\left(\mathrm{CO}_{2}\right)$ species. Coupled with MOEMS architecture, miniature and cost effective $\mathrm{CO}_{2}$ sensors can be developed using this laser diode technology for use in localized environments, short range Differential lidar applications as well as static sensors for tracking gas clouds on Martian environment. Static sensors could be configured as distributed point sensors based network on Martian environment.

Waveguide Laser Components: Waveguide laser for operation at $1064 \mathrm{~nm}$ wavelength include an high power $808 \mathrm{~nm}$ pump diode bar, laser gain medium, a saturable absorber, and dielectric mirrors. Waveguide laser is an emerging technology that offers several unique features. Compared to other solid-state laser technologies, such as fiber lasers, the self-imaging waveguide offers higher peak and average power performance, and superior thermal waste heat management. As such, they are attractive as reliable, stable sources for imaging and high bit rate, high power optical communications. The unique, patented waveguide gain media ${ }^{5}$ offers additional capabilities for space applications, such as amplifiers and pump sources for nonlinear optical devices. The laser diodes will provide risk reduction data for producing higher mean time between failure (MTBF) devices. The optical components that are proposed for space exposure testing are relevant to laser communication, laser range finding, hyperspectral imaging, and vibrometry applications. Furthermore, waveguide based lasers would be suitable for expanding future space communication networks, proximity operations, and guidance, navigation and control.

\subsection{Optical elements on a specimen holder}

Figure 1 shows the optical items mounted on a specimen holder. The laser diode, diode bar, ceramic yttria and Bismuth doped $\mathrm{PbTe}$ are visible. Other elements are below a secondary plate and are hidden from direct view. This specimen holder was mounted on MISSE 6B. Figure 2 shows PEC mounted on a carrier with all other specimen trays. Figure 3 illustrates fully integrated PEC on a test platform. 

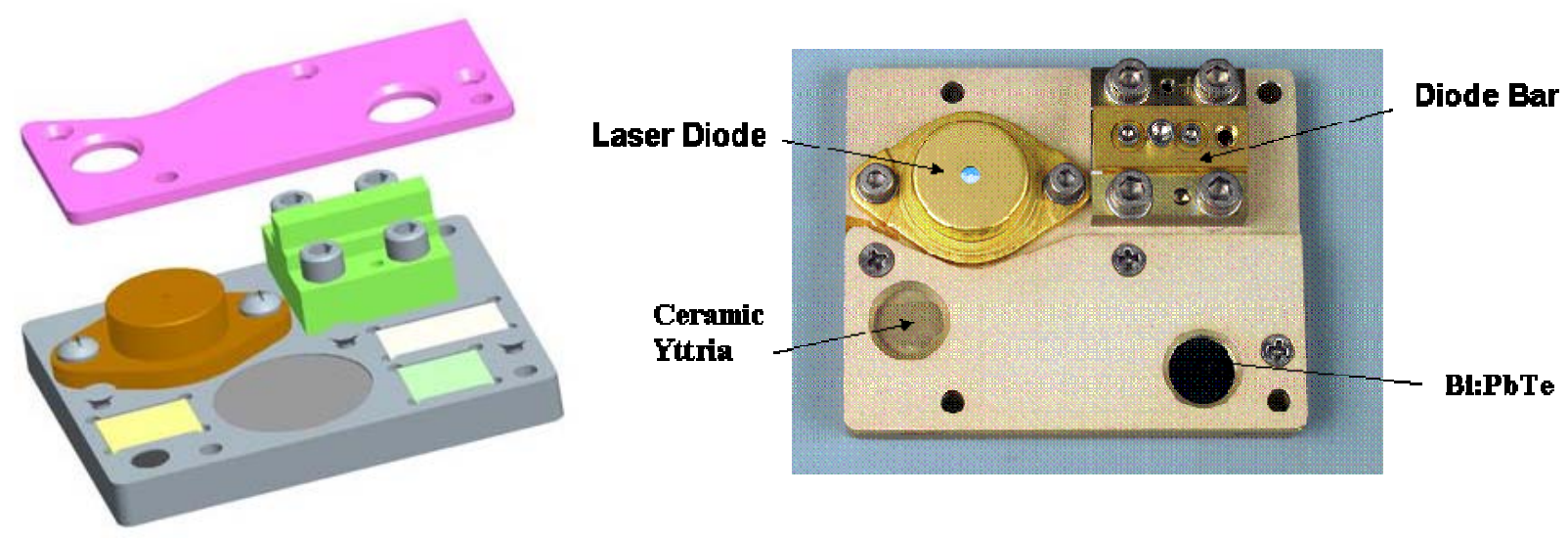

Fig. 1. The optical elements rigidly mounted in a specimen holder.

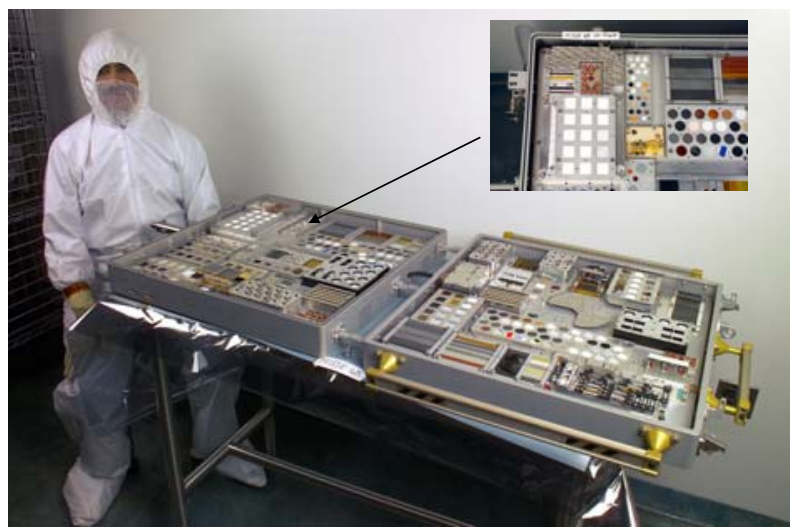

Fig. 2. The specimen holder is integrated inside the PEC with carrier. The figure insert on top right shows the location of our specimen holder.

Fig. 3. The PEC with carrier on a test platform.

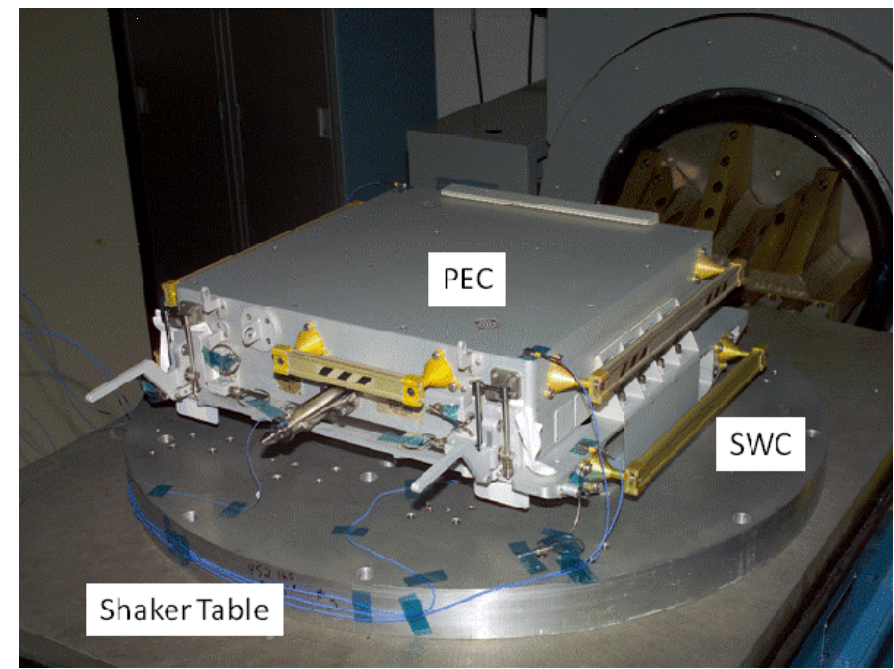




\section{ENVIRONMENTAL TESTS}

MISSE 6A and 6B are PECs each housing active and passive experiments to be exposed in the space environment for characterization. Conforming to NASA procedures, a test plan was drawn up for dynamic verification of the MISSE 6 integrated PECs. Quality assurance provisions were in place for carrying out environmental test procedures. The components were mounted on a pair of trays fastened inside PECs in an anti-static clean room. The PECs were securely stored for follow-on environmental tests. Two sidewall carriers (SWC) attached to the PECs aided in safe handling of the unit. The SWCs are used for stowing PECs in the Shuttle Bay for launch and return. The increased Capability Adaptive Payload Carriers (ICAPC) are used for mounting each of the SWCs to the Shuttle Cargo bay sidewall. The test specimens were attached to a shaker system capable of allowing vibration measurements along $\mathrm{X}, \mathrm{Y}$ and $\mathrm{Z}$ axes. The shaker unit is equipped with computer controlled data acquisition and control system for controlling all aspects of the test. Shaker control acceleration and measurement accelerometers were routed through appropriate signal conditioning to the data acquisition system ${ }^{6,7}$.

Dynamic environment tests were carried out with the integrated PECs stowed in its respective SWC to simulate the interface with the ICAPC in the Orbiter cargo bay. The main objective of the dynamic test plan is to perform a series of vibration tests to qualify the MISSE PECs for flight in its Orbiter sidewall mounting configurations. This includes verifying that the PECs can withstand acceptance launch vibration levels for the Shuttle Orbiter, and verifying that the first mode frequency of the test specimen is greater than $35 \mathrm{~Hz}$. The PECS were subjected to a series of sine sweeps and random vibration tests. The sine sweeps were used as a part of nondestructive evaluation (NDE) of the test specimens. The test sequence consisted of baseline pre-random, random, and post random sine sweeps were performed along one axis at a time until all three axes were tested and appropriately documented. For e.g., the vibration levels for the sine sweep consisted of a $0.25 \mathrm{~g}$ upward sweep from 10 to $800 \mathrm{~Hz}$ at a sweep rate of 2 Octaves/min, followed by a $0.25 \mathrm{~g}$ downward sweep from 800 to $10 \mathrm{~Hz}$ at a sweep rate of 2 Octaves/min. For sine sweep testing, control and response accelerometer power spectral densities were acquired. In the case random vibration tests, control and response power spectral densities were acquired.

The tests results were compared in each case to verify that the test specimen was not damaged as a result of the high level random vibration tests. The results confirmed that the fundamental dynamics of the structure were not changed due to high-level random vibration tests.

\section{DEPLOYMENT OF MISSE 6}

The MISSE 6 was transported on STS-123 Mission to ISS on March 11, 2008 after the envirornmental tests ere sucessully completed. On Tuesday, March 18, 2008, during the mission's third spacewalk, the Mission Specialist Rick Linnehan attempted to install the MISSE 6 experiment onto the Columbus module. This attempt failed because latching pins used to hold the experiment packages onto the hull of Columbus could not be properly engaged ${ }^{8}$. Following five spacewalks, astronauts Robert L. Behnken and Mike Foreman completed the final spacewalk of the STS-123 mission at 9:36 p.m. CDT. Their 6-hour, 2-minute EVA was highlighted by the installation of the MISSE-6. Behnken installed the MISSE-6 on the outside of the Columbus laboratory. With the help of a few troubleshooting methods developed by engineers on the ground, Behnken successfully installed MISSE- 6 onto the Columbus module. Figure 4 shows the MISSE 6 PEC attached to the Columbus module on the ISS. Figure 5 illustrates the orientation of MISSE 6 along with the velocity vector as seen from the STS-123.

\section{RETRIEVAL OF MISSE 6 PEC}

The MISSE 6 PEC is anticipated to be retrieved on STS-128 mission in August 2009. Following laboratory tests, a detailed comparative analysis of the above mentioned specimens will be carried out to understand the space effects. The results will be reported in upcoming conferences. 

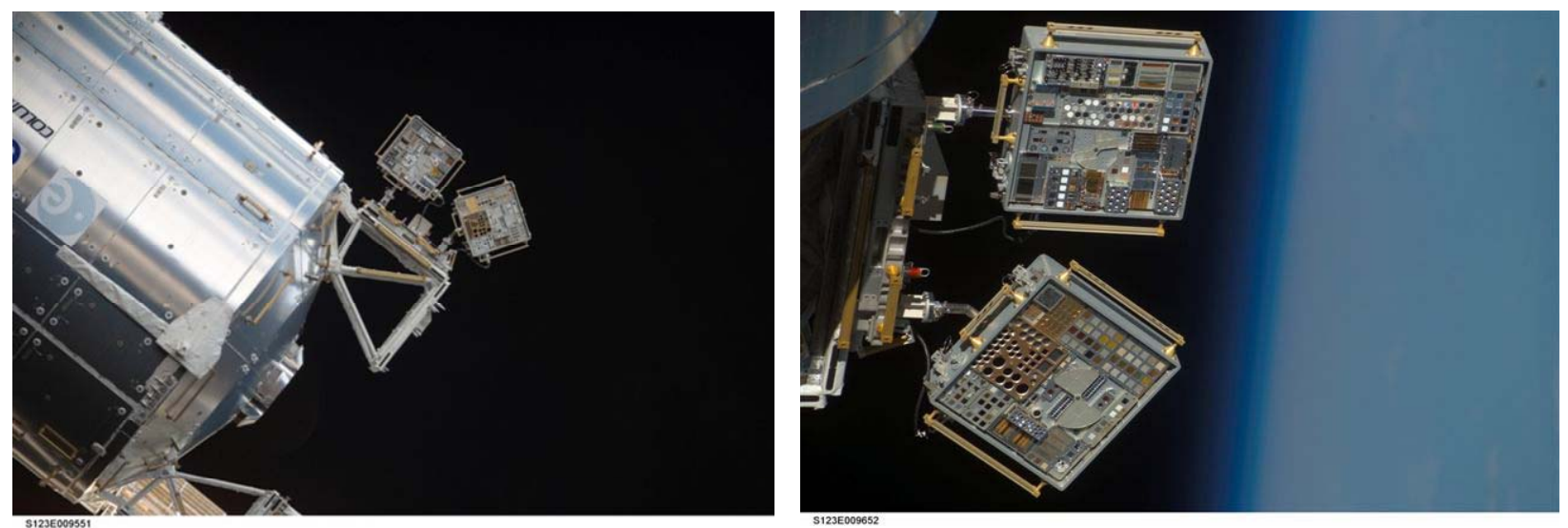

Fig. 4. (Left) The PEC attached to the Columbus module on ISS. (Right) A close-up view of MISSE PEC in space.

\section{SUMMARY AND CONCLUSIONS}

The objective of the MISSE program is to study the performance, stability, and long-term survivability of novel materials when subjected to the synergistic effects of the harsh space environment. So far, five MISSE missions have been completed. The MISSE 6 mission was launched on March 11, 2008. Several optical specimens including waveguide laser components, laser diodes, thermoelectric power generator materials and ceramic yttria specimens were successfully placed on the ISS for primarily radiation and UV exposure. These specimens will be retrieved in 2009. Upon retrieval, these specimens will undergo similar tests to look into any possible deviations from pre-exposure characteristics.

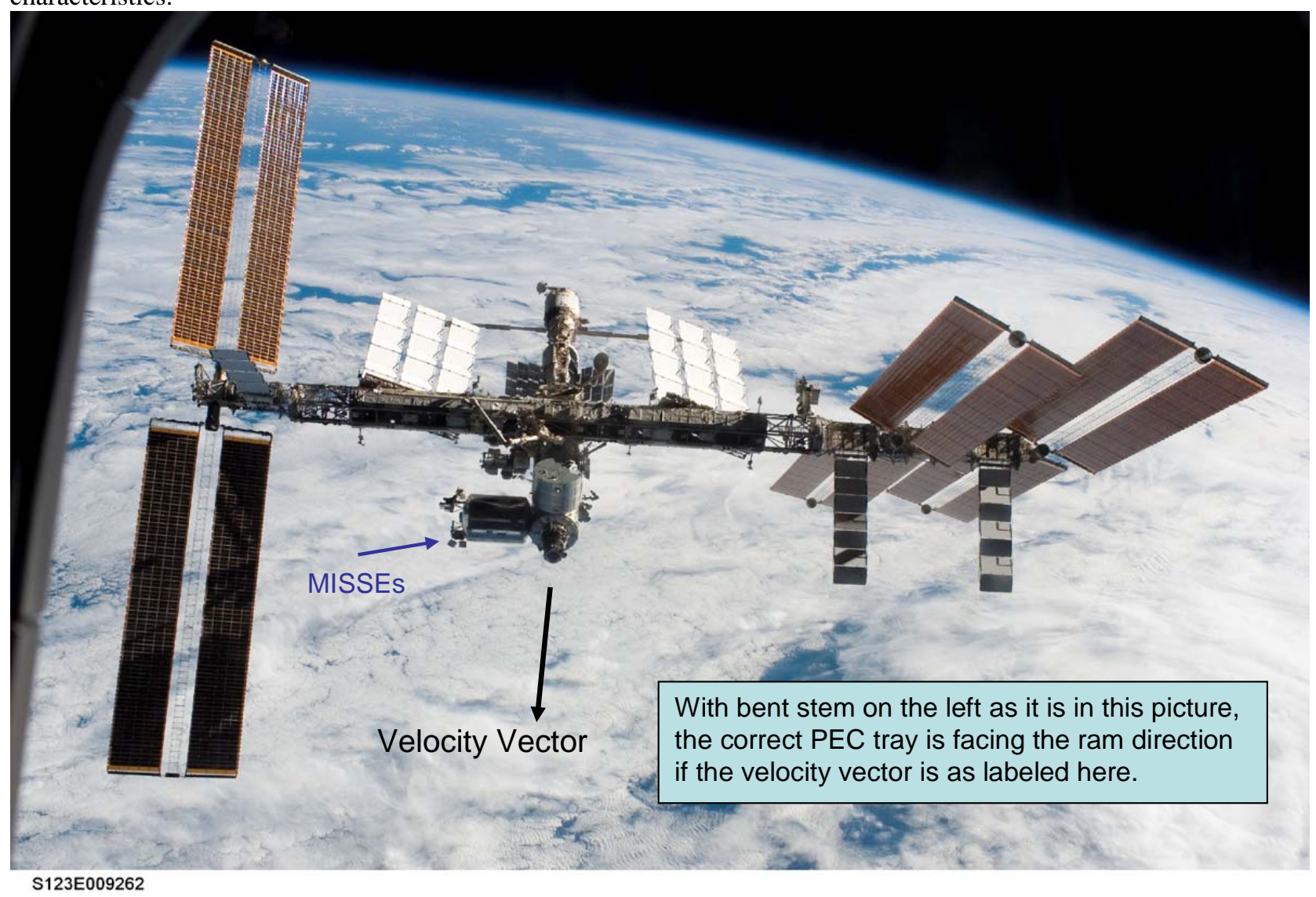

Fig. 5. Orientation of MISSE 6 PEC on ISS. 


\section{REFERENCES}

[1] William H. Kinard, “MIR Environmental Effects Payload (MEEP) Archive System,” NASA, Langley Research Center, Hampton, Virginia. (http://setas-www.larc.nasa.gov/meep/meep.html and http://www.nasa.gov/centers/langley/news/factsheets/misse_2005.html).

[2] The NASA MISSE website: http://misseone.larc.nasa.gov/.

[3] Narasimha S. Prasad and William H. Kinard, “MISSE 6-Testing Materials in Space," Proc. SPIE 7095, 7095OD (2008).

[4] Prasad, N.S., Edwards, W.C., Trivedi, S.B.. Kutcher, S.W., Chen-Chia Wang, Joo-Soo Kim, Hommerich, U., Shukla, V., Sadangi, R., Kear, B.H., "Recent Progress in the Development of Neodymium-Doped Ceramic Yttria," Selected Topics in IEEE Journal of Quantum Electronics, Vol. 13, Issue 3, 831 - 837(2007).

[5] US Patent Number 6,894,828, “Power scalable waveguide amplifier and laser device” (2005).

[6] Justin D. Templeton and Chad E. Rice, "Vibration test procedure for the MISSE PEC 6B in the SWC," NASA document No. MISSE 6-088.

[7] Justin D. Templeton and Chad E. Rice, "Vibration test procedure for the MISSE PEC 6B in the SWC," NASA document No. MISSE 6-089.

[8] STS-123 MCC Status Report \#15 (http://www.nasa.gov/mission_pages/shuttle/shuttlemissions/sts123/news/STS123-15.html).

[9] STS-123 MCC Status Report \#25 (http://www.nasa.gov/mission_pages/shuttle/shuttlemissions/sts123/news/STS123-25.html). 